\title{
Internal to External Microfluidic Device for Ellipsometric Biosensor
}

\section{Application}

\author{
Valdemar Stankevič ${ }^{* 1,2}$, Tomas Rakickas ${ }^{1}$ and Gediminas Račiukaitis ${ }^{1}$ \\ ${ }^{1}$ Center for Physical Sciences and Technology, Savanoriu Ave. 231, LT-02300, Vilnius, Lithuania \\ E-mail: valdemar.stankevic@ftmc.lt \\ ${ }^{2}$ ELAS UAB, Savanoriu Ave. 231, LT-02300, Vilnius, Lithuania
}

\begin{abstract}
The femtosecond pulses offer a flexible way to induce internal modifications inside fused silica and prepare embedded 3D microchannel structures. In this paper, an internal to external microfluidic device is presented. The micro-channels were prepared with the direct laser writing technique using different scanning methods. The sample was immersed in diluted $10 \%$ concentration HF acid to remove the modified material. A few independent $3 \mathrm{D}$ microchannels were inscribed, and each of them was connected to a pump to circulate the protein solution during the measurement preserving a constant flow rate. Microchannels were designed to act as flow cells in ellipsometric imaging surface plasmon resonance (iSPR) setup to study the real-time interaction kinetics of biological materials (proteins or DNA). The microchannels allow to make few parallel measurements at the same time and minimize the required biological sample amounts for the iSPR experiment.
\end{abstract}

DOI: $10.2961 /$ jlmn.2016.01.0010

Keywords: Femtosecond, microchannel, chemical etching, iSPR, parallelization

\section{Introduction}

Attractive properties of microfluidic devices like the integration of different and complex processes on a single chip, ease of operation, small sample and reagent consumption, quick analysis, and others [1] made them useful in a broad area of applications, particularly in medical diagnostics and biology research $[2,3]$.

Many polymers were used to fabricate microfluidic devices. Very popular is optically transparent, soft elastomer polydimethylsiloxane (PDMS). However, it is permeable to many small and hydrophobic molecules [4]. Microchannels in PDMS are typically made using soft lithography [5]. This technique can be cheap and very flexible, but the fabrication of the $3 \mathrm{D}$ microfluidic structures in the transparent substrate requires additional stacking and bonding processes that increase the total fabrication time [6]. An alternative promising technique to fabricate the fully integrated $3 \mathrm{D}$ structures is a femtosecond laser irradiation followed by chemical etching (FLICE) of transparent glass materials that was reported first by Marcinkevičius et al. [7] and received strong development in the past few decades $[8,9$, $10,11]$. Fused silica is the main transparent media in this technique due to its easy processing and high etching selectivity caused by the type II modification $[12,13]$. The optical quality, chemical resistivity and flexibility of the FLICE technique are the key factors that stimulated the use of fused silica in this work. The FLICE technique involves inscribing of 3D microchannels in few steps that do not require any complex fabrication processes.

In this paper, an internal to external microfluidic device is presented. Microchannels were prepared with the direct laser writing technique using different scanning methods [14]. A sample was immersed in diluted $10 \%$ concentration $\mathrm{HF}$ acid to remove the modified material.
Microchannels were designed to act as flow cells [15] in the imaging ellipsometric surface plasmon resonance (iSPR) setup to study the real-time kinetics of biological materials (proteins or DNA). Most of the current iSPR devices [16-18] measures the data from a single channel that makes the measurement process time consuming and expensive. Typically one sensor chip is used for a single measurement only and for the next measurement the new sensor chip should be used. Additionally, the flow cell has a finite volume that could be in the range of $100 \mu 1$ or more. Therefore, it can take up to few minutes for new injection solution to mix and replace the previous one. The goal of this study is to prepare and test an array of separate parallel microchannels in fused silica with minimized flow cell volumes suitable for iSPR measurements. Set of separate parallel microchannels were inscribed, and each of them was connected to the pump to circulate the protein solution during the measurement preserving a constant flow rate. This microchannel configuration due to parallelization of independent measurements and decreased solution mixing times permits to obtain a few protein association and dissociation kinetics on the same sensor chip by shifting the microchannel position.

\section{Experimental}

\subsection{The micromachining setup}

The modification inside fused silica for microfluidic device presented in this paper were prepared with Yb:KGW femtosecond laser (Pharos, Light Conversion Ltd.) with a pulse duration of $360 \mathrm{fs}$ working at $515 \mathrm{~nm}$ wavelength. The pulse repetition rate of the laser was varied in the range of $100 \mathrm{kHz}-500 \mathrm{kHz}$ and the maximum average laser power of $6 \mathrm{~W}$. The average laser 
power was attenuated by motorized attenuator during the fabrication process.

The experimental setup is shown in Fig. 1. The beam passed the $2 \mathrm{x}$ demagnification lens system composed of two lenses in Galilean configuration (focal length of $100 \mathrm{~mm}$ and $-50 \mathrm{~mm}$ ). Then laser beam passed through an aperture stop and focusing objective (Olympus LMPlan, NA 0.55). The spot diameter at the focal plane was $<2 \mu \mathrm{m}$ at $1 / \mathrm{e}^{2}$. Linear positioning stages (Aerotech ANT 130-160 $\mathrm{XY}$ ) that allow $<1 \mu \mathrm{m}$ accuracy were used for sample translation. The off-axis visualization system was used for precise laser beam positioning on the sample surface.

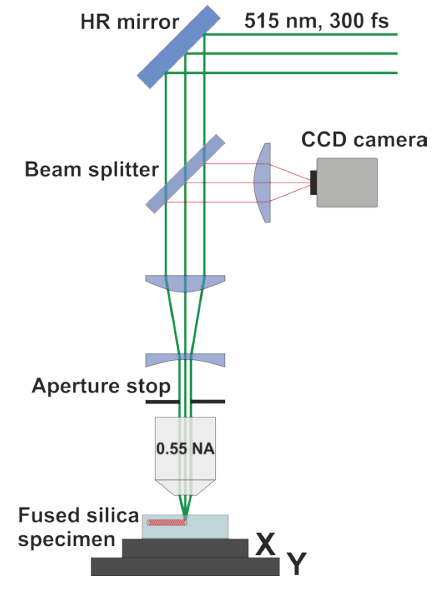

Fig. 1 The experimental setup for laser direct writing of microfluidic channels.

The microfluidic devices were formed in commercially available optically-polished ultraviolet-grade fused silica sample (JGS1) with $20 \times 15 \times 2 \mathrm{~mm}^{3}$ dimensions. The workpiece was placed on a vacuum chuck to keep the stable sample position during the high acceleration movement of positioning stages. The translation speed ranging from $1 \mathrm{~mm} / \mathrm{s}$ to $20 \mathrm{~mm} / \mathrm{s}$ was selected to find the optimal microchannel formation conditions. The polarization was set to linear and perpendicular to the translation direction during the whole experiment.

\subsection{Imaging surface plasmon resonance setup}

Imaging surface plasmon resonance experiments were performed in Kretschmann configuration [19] with imaging null-ellipsometer (nanofilm_ep3se, Accurion $\mathrm{GmbH}$ ). The setup is based on total internal reflection ellipsometry [20], using the null ellipsometer, microscope objective to focus the light and CCD camera as a detector. Gold coated BK7 glass sensor chips (Biacore) and $60^{\circ} \mathrm{BK} 7$ glass prism were used. Measurements were carried out with a $658 \mathrm{~nm}$ wavelength laser light at fixed angle of incidence that was $0.1^{\circ}$ below SPR resonance angle. In our case, the angle of incidence was $70.2^{\circ}$. The ellipsometric parameters $\Delta$ and $\Psi$, averaged over selected regions of interest, were recorded over time at $20 \mathrm{~s}$ intervals. With this set of parameters, $\Delta$ was inversely proportional to mass density increase on the sensor chip surface. For thickness modelling, the included data analysis program, EP4 Model was used.

For all biosensor experiments and buffer preparation, the ultrapure water was used, taken directly from Synergy
UV water purification system (Merck Millipore). Before its use, the sensor chips were cleaned with $\mathrm{SC}-1$ solution, a mixture of ultrapure water, $30 \%$ hydrogen peroxide (Carl Roth $\mathrm{GmbH}$ ), and 25\% Ammonia solution (Carl Roth $\mathrm{GmbH}$ ) in a ratio of $5: 1: 1$, respectively, at $85^{\circ} \mathrm{C}$ for $7 \mathrm{~min}$, two times, followed by excessive rinsing in ultrapure water and drying with a nitrogen gas stream.

The self-assembled monolayer was formed by incubating the $\mathrm{SC}-1$ cleaned sensor chip overnight in a solution of thiols in ethanol (Sigma-Aldrich), containing $8 \mu \mathrm{M}$ concentration biotin functional group containing thiol (HS- $\mathrm{C}_{11}-\mathrm{EG}_{3}$-biotin, ProChimia) and $32 \mu \mathrm{M}$ concentration protein adsorption resistant $\mathrm{EG}_{3}-\mathrm{OH}$ group terminated thiol (HS- $\mathrm{C}_{11}-\mathrm{EG}_{3}-\mathrm{OH}$, ProChimia). After incubation, the sensor chips were rinsed and sonicated in ethanol for 3 min twice, sonicated in ultrapure water and dried under a nitrogen gas stream.

\section{Formation and investigation of internal to external microchannels}

To get the internal to external microchannel, a part of the modified zone of fused silica should be near the sample surface, and the top part of the channel appears as surface damage. The design of such microfluidic channel is presented in Fig. 2. The microchannels were inscribed using a direct laser writing technique. The 3D model of the microchannel was imported to the fabrication program (SCA, Altechna R\&D) and sliced into planes with the distance between planes equal to $5 \mu \mathrm{m}$. Each plane was hatched by lines, and the scanning direction of lines was perpendicular to the laser beam polarization. The scanning speed and laser pulse energy were as follows: $4.5 \mathrm{~mm} / \mathrm{s}$ and $400 \mathrm{~nJ}$. For the first etching approach, the modified sample was submerged in dilute $10 \%$ concentration $\mathrm{HF}$ acid for 12 hours. Improvement in the etching time was achieved by immersing a jar with the acid in an ultrasonic water bath at $40{ }^{\circ} \mathrm{C}$. In this case depending on the etching conditions, the etching rate was increased from 5 to 10 times.

At the outer part of a microchannel, the large area of the modified zone is exposed to etching solution (along the all external part of the microchannel). In this case, etching goes in the perpendicular direction to the microchannel direction. However in the internal part of the microchannel, only the cross-section of the microchannel is open for acid, and the etching goes along the embedded modified zone. Therefore, the external part of the microchannel is formed much faster than the internal part. According to our investigations, the maximum achieved etching rate in $10 \%$ $\mathrm{HF}$ for fused silica modified by $500 \mathrm{kHz}$ and $400 \mathrm{~nJ}$ pulses was $\sim 1000 \mu \mathrm{m} / \mathrm{h}$ while for unmodified fused silica $15 \mu \mathrm{m} / \mathrm{h}$ (see below). This gives the etching selectivity of $\sim 70$ times. To fabricate good-quality microchannels, the etching selectivity should be taken into account. The modification depth for external microchannel is $\sim 40 \mu \mathrm{m}$ that finishes on the surface by the surface ablation. The modification width of the microchannel is $50 \mu \mathrm{m}$ for all microchannel length. Taking into account the $1000 \mu \mathrm{m} / \mathrm{h}$ etching rate of laser modified zone, the estimated etching time for external part of the microchannel is only $\sim 3 \mathrm{~min}$., and the rest of time, the width of the external microchannel increases with the $15 \mu \mathrm{m} / \mathrm{h}$ rate (Fig. $4 \mathrm{f}$ ). The depth of the external part of microchannel after 1 hour of etching re- 
mains almost unchanged. The length of embedded part of the microchannel varied from 2.5 to $3.5 \mathrm{~mm}$, and the etching of this part took place from both sides (from the transition between the external and embedded part and from the connector side), this part was fully etched after $\sim 75 \mathrm{~min}$. $-105 \mathrm{~min}$. This estimation shows that the design of external to internal microchannels should be with minimized length of embedded microchannel length to save the outer part of the microchannel from degradation.

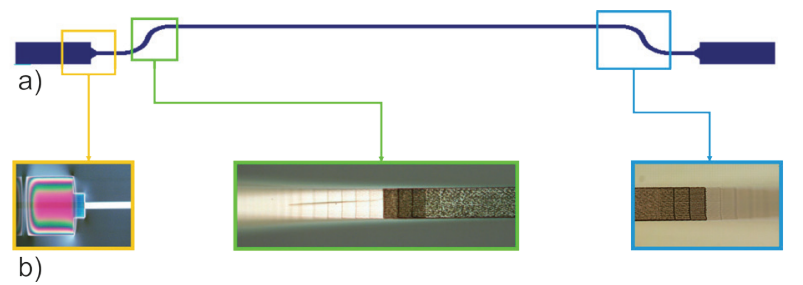

c)

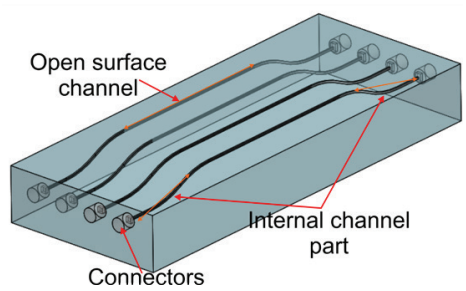

Fig. 2 The sketch design of microfluidic semi-internal microchannel (a). The optical microscope pictures from the top of the laser-induced modifications in fused silica (b) and the setup of internal to external microchannels design (c).

The design of microchannels plays a significant role for the etching time and quality of the external microchannel part. The external part of microchannels was used as a flow cell for iSPR measurements while internal part was used to deliver the fluids to the external part and connect the external tubing. A few designs of microchannels were prepared. The first design was used only to check the functionality of the single channel measurements (Fig 2 a). The second design was developed with close to each other placed microchannels to allow parallel measurements (Fig. 3 a).

a)
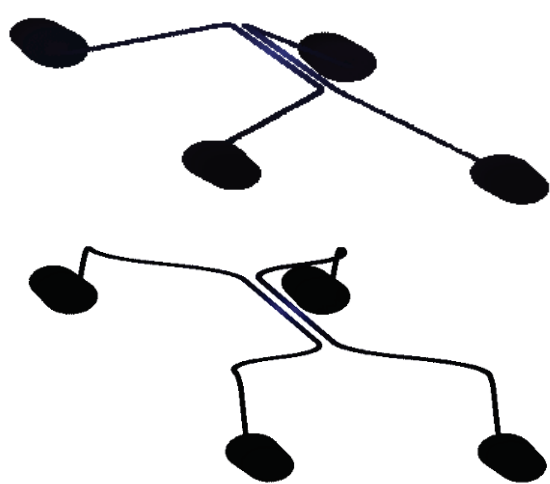

b)

Fig. 3 The optimized design of the internal to external microchannel: (a) second design approach; (b) the last design approach. The vertical dimensions are scaled by the refractive index of fused silica.

The latest design was developed with a close distance of adjacent microchannels and the minimized length of the embedded part of the microchannel (Fig 3 b). As the internal part of the microchannel is embedded in fused silica, its vertical dimensions should be scaled by the refractive index of fused silica $(\mathrm{n} \sim 1.4615$ at $515 \mathrm{~nm})$ to get the correct shape of the microchannel due to the light refraction in transparent materials.

Following the described design, the samples were fabricated and etched in 10\% HF acid in an ultrasonic bath for 2 hours. To get the microchannels with different surface roughness, each microchannel was fabricated with various fabrication parameters. The fabricated microchannels with different design approaches are shown in Fig. 4. The fabrication parameters of microchannels shown in Fig. 4c are $300 \mathrm{~nJ}, 10 \mathrm{~mm} / \mathrm{s}, \mathrm{dy}=3 \mu \mathrm{m}$ and $\mathrm{dz}=5 \mu \mathrm{m}$ for the left channel in Fig. $4 \mathrm{c}$ and $200 \mathrm{~nJ}, 5 \mathrm{~mm} / \mathrm{s}$, dy $=1.5 \mu \mathrm{m}$ and $\mathrm{dz}$ $=5 \mu \mathrm{m}$ for the right microchannel. In Fig $4 \mathrm{e}$, the fabrication parameters are $300 \mathrm{~nJ}, 5 \mathrm{~mm} / \mathrm{s}$, dy $-3 \mu \mathrm{m}$ and $\mathrm{dz}-3 \mu \mathrm{m}$ for the left channel and $300 \mathrm{~nJ}, 10 \mathrm{~mm} / \mathrm{s}$, dy $=$ $3 \mu \mathrm{m}$ and $\mathrm{dz}=5 \mu \mathrm{m}$ for the right microchannel. Influence of the processing parameters to the surface roughness will be described in the next chapter. Due to the longer external part of the microchannel in third design, the etching time was minimized to 2 hours.

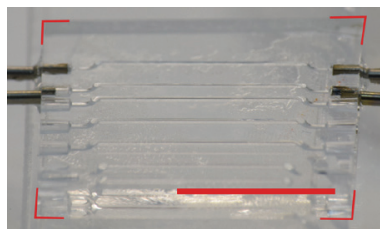

a)

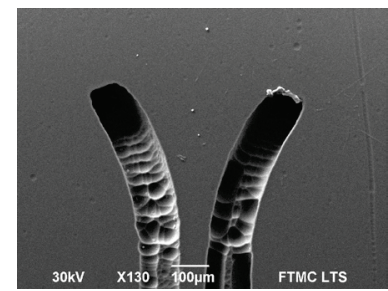

c)

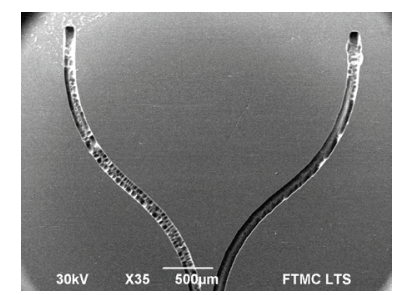

e)

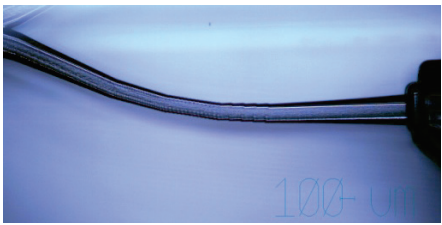

b)

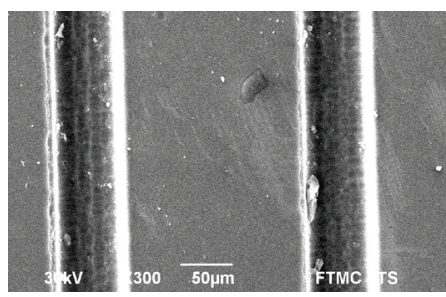

d)

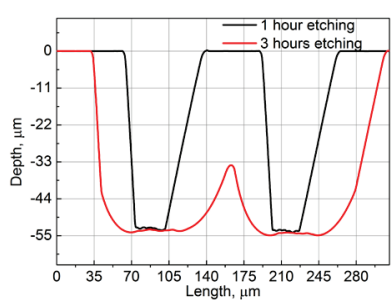

f)
Fig. 4 The picture of fabricated microchannels with different design approaches: a) full microchannel set with integrated connection tubes prepared for testing. The red scale bar indicates $10 \mathrm{~mm}$; b) Side view of the embedded microchannel part etched for 12 hours in $10 \% \mathrm{HF}$ acid; c) SEM picture of the transition from the external to internal microchannel with the second design approach etched in HF acid 2 hours; d) The top view of microchannels of the second design approach; e) SEM picture of the transition from the external to internal microchannel with the third design approach etched in HF acid for 2 hours; f) The depth profile of microchannels in Fig 4 d. after different etching time.

From the depth profile of microchannels in the second design, the etching rate of non-modified fused silica can be estimated, and it is $\sim 15 \mu \mathrm{m} / \mathrm{h}$. The volume of the etched microchannel increased, however, the depth remained al- 
most unchanged because the measurements of depth were done with respect to sample surface that was also etched with the same $\sim 15 \mu \mathrm{m} / \mathrm{h}$ etching rate.

\subsection{Surface roughness of external channel part}

During the ellipsometric measurements, the solution of proteins flows through the external part of the microchannel, and the measurement results depend on the quality of the microchannel because of flow disturbance and light scattering from the channel walls. The surface roughness of the external microchannel was investigated. For that purpose, an array of external microchannels was fabricated varying the pulse energy $(200 \mathrm{~nJ}-400 \mathrm{~nJ})$ and scanning speed $(2.5 \mathrm{~mm} / \mathrm{s}-20 \mathrm{~mm} / \mathrm{s})$. The processing parameters for each set are shown in Table 1.

Table 1 The processing parameters of external channels

\begin{tabular}{|c|c|c|c|c|}
\hline $\begin{array}{l}\text { Microchannel } \\
\text { set nr. }\end{array}$ & $\begin{array}{l}\text { Laser pulse } \\
\text { energy for } \\
\text { one set, nJ }\end{array}$ & $\begin{array}{l}\text { dy, } \\
\text { um }\end{array}$ & $\mathrm{dz}, \mathrm{um}$ & \begin{tabular}{ll}
\multicolumn{2}{l}{ Translation } \\
speed for \\
one set, \\
$\mathrm{mm} / \mathrm{s}$
\end{tabular} \\
\hline 1 & \multirow{5}{*}{$200-400$} & 3 & 5 & \multirow{5}{*}{$2.5-20$} \\
\hline 2 & & 1.5 & 5 & \\
\hline 3 & & 5 & 5 & \\
\hline 4 & & 5 & 3 & \\
\hline 5 & & 5 & 7 & \\
\hline
\end{tabular}

One channel was inscribed by a set of scans in the ydirection with the distance between scans $-d y$ and in the zdirection with the distance between scans - $d z$. Five sets of the microchannel arrays with different $d y$ and $d z$ distances were fabricated. The last layer was focused on the sample surface to initiate the surface ablation.

The fabricated external channels were immersed into dilute $10 \%$ concentration $\mathrm{HF}$ acid, placed into the ultrasonic bath and etched for 1 hour. After one hour, the sample was inspected with an optical microscope (Olympus), scanning electron microscope (JEOL JSM6490LV) and stylus profiler (Bruker Dektak 150+) to measure the longitudinal surface roughness of the etched channels. Then it was placed back for etching for one more hour. After 2 hours of etching, the sample was inspected with the same methods.

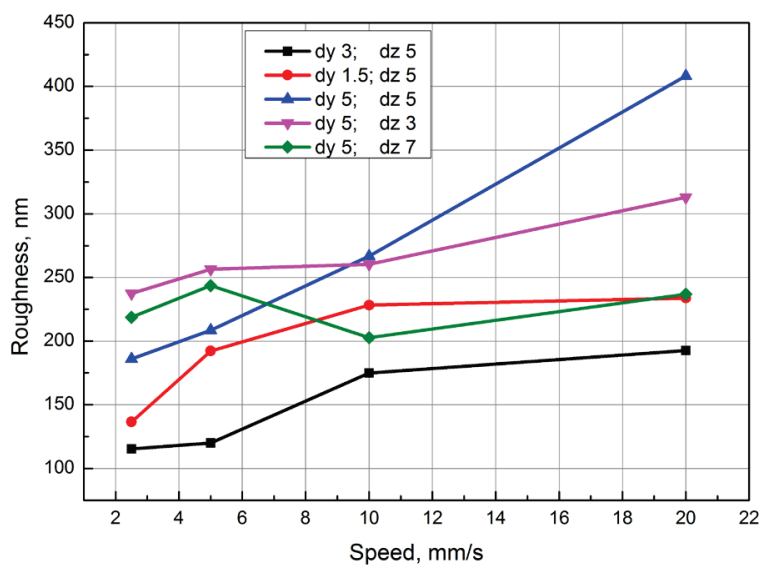

Fig. 5 Dependence of longitudinal roughness of the external channel part on the scanning speed. The microchannel etching time in $\mathrm{HF}$ acid was 2 hours. The laser pulse energy and repetition rate were set as constant: $200 \mathrm{~nJ}$ and $500 \mathrm{kHz}$.
The surface roughness dependence on the scanning speed using the constant $200 \mathrm{~nJ}$ laser pulse energy is shown in Fig. 5. The surface roughness decreased reducing the scanning speed. More pulses were delivered to a single point with the lower scanning speed at a constant pulse energy. Therefore, the overlap of adjacent modifications increased and larger exposure dose to single point induced stronger modification of surrounding material. That led to the smaller grain size, and smaller surface roughness was achieved. The SEM pictures of the etched microchannel prepared using different processing energy are shown in Fig 6 . The grain size increased by increasing the pulse energy that coincides with the results reported in the previous work [14].

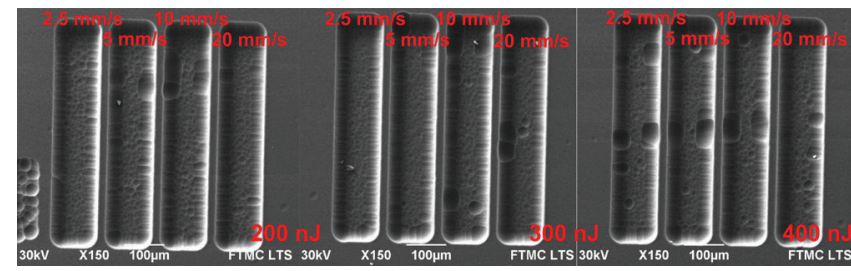

Fig. 6 Images of external microchannels etched for 2 hours in $10 \% \mathrm{HF}$ acid.

\section{Assembling and characterization of microfluidic device}

\subsection{Assembling}

First of all, the microfluidic device was connected with the prepared sensor chip - glass substrate coated with a thin gold layer. The non-modified surface of the microfluidic device was also affected by HF acid. Therefore, the flatness was not sufficient to ensure hermetical sealing between the external part of the microchannel and the Au-coated sensor chip. To secure the reliable sealing, a thin PDMS interlayer with the thickness of $100 \mu \mathrm{m}$ was added. The fluid to the sensor chip surface was delivered through the microchannel size slits in PDMS interlayer. The slits in PDMS were cut by the laser. After laser cutting, the PDMS interlayer was cleaned in ethanol and mounted on the top of the device with open microchannels. Then the glass surface was hermetically sealed with the PDMS layer. For the final step, a prism was placed on the sensor chip to obtain the Kretschmann configuration for iSPR setup. The design of assembled iSPR setup is shown in Fig 7.

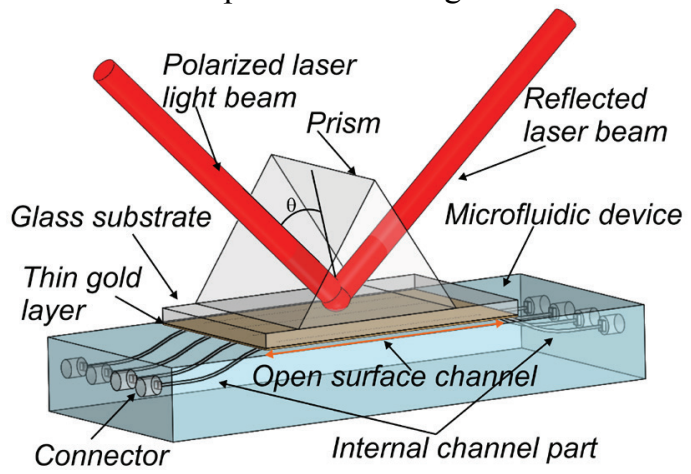

Fig. 7. The design of iSPR setup with the integrated internal to the external microfluidic device. The fabricated microchannels were covered with the thin PDMS interlayer and the gold-coated glass substrate (sensor chip). A prism was mounted on the sensor chip. 


\subsection{Characterization}

To test the performance of the assembled microfluidic device, it was connected to the constant flow rate peristaltic pump which pumped the HEPES buffer $(20 \mathrm{mM}$ HEPES, $150 \mathrm{mM} \mathrm{NaCl}, \mathrm{pH} \mathrm{7.5)}$ at $150 \mu \mathrm{l} / \mathrm{min}$ flow rate from vial A. The fully-integrated and connected device was mounted into the ellipsometer for the specific protein adsorption kinetic measurement (Fig. 8).

The specific and well-known biotin - streptavidin recognition reaction [21] was chosen to test the system performance. The sensor chips were modified with biotin functional group bearing self-assembled monolayer.

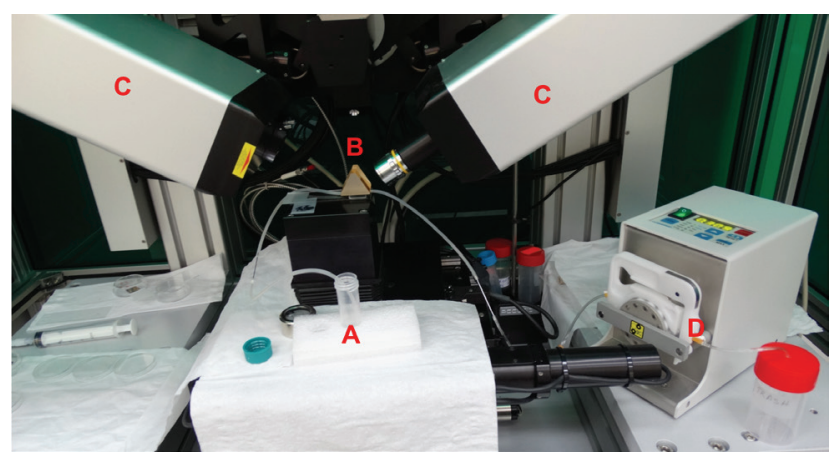

Fig. 8. The fully integrated microfluidic device for ellipsometric measurements. A - vial with a buffer fluid and specific protein, B - the microfluidic device with the mounted prism, $\mathrm{C}$ - ellipsometer, D - fluid pump.

After rinsing with the buffer, $1 \mathrm{mg} / \mathrm{ml}$ bovine serum albumin (BSA, Sigma-Aldrich) solution in HEPES was injected for $5 \mathrm{~min}$. No protein adsorption was visible, what indicated that the sensor surface was sufficiently protein resistant (Fig 9). The second injection was the protein that should adsorb specifically on biotin groups - streptavidin. We injected $250 \mathrm{nM}$ concentration streptavidin (Serva Electrophoresis $\mathrm{GmbH}$ ) solution in the buffer for $25 \mathrm{~min}$. The specific adsorption was clearly visible. The thickness of adsorbed streptavidin layer according to the ellipsometric model calculations was $0.2-0.3 \mathrm{~nm}$. The break in the kinetics appeared because of the air bubble, trapped in the microchannel.

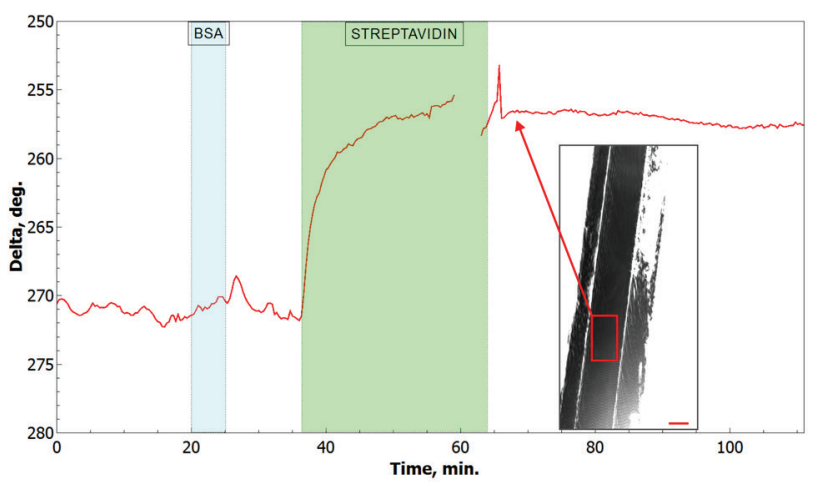

Fig. 9. The specific protein adsorption kinetic measurement results achieved in the fabricated microfluidic device. Abbreviation on the graph: BSA - bovine serum albumin. Inset - the measurement zone in the microfluidic channel that is seen in the ellipsometric device. The red scale bar indicates $50 \mu \mathrm{m}$.

\section{Conclusion}

In conclusion, the internal to external microchannels were fabricated and integrated into a single device for the imaging ellipsometric surface plasmon resonance setup. A few design approaches were investigated. It is evident, that to improve the etching time the length of embedded microchannel part should be minimized. The minimal length of the internal microchannel is restricted by the length of connector that should be $\geq 1 \mathrm{~mm}$ in order to ensure the qualitative connection and transition from embedded channel part to the external part. The roughness investigation of the external microchannel part showed that the processing with lower pulse energy $(200 \mathrm{~nJ})$ and low speed $(<10 \mathrm{~mm} / \mathrm{s}$ at $500 \mathrm{kHz}$ ) should be performed to improve the quality of microchannel. However, to optimize the processing time, scanning speed $\geq 10 \mathrm{~mm} / \mathrm{s}$ should be applied, that results in a slight decrease of the external channel quality.

The microfluidic device was completely assembled, and the protein adsorption kinetic was measured. The formed parallel microchannels allow the measurement parallelization that enables more efficient use of sensor chip and repeatability of experimental conditions. Due to decreased volumes of flow cells, much shorter solution mixing time was obtained, that made it possible the registration of the protein association kinetics at a constant its concentration.

\section{Acknowledgments}

Dr. Ramūnas Valiokas (CPST) is acknowledged for technical support in surface modification/iSPR technology.

\section{References}

[1] E. K. Sackmann, A. L. Fulton, and D. J. Beebe, Nature, 507, (2014) 181.

[2] P. Yager, T. Edwards, E. Fu, K. Helton, K. Nelson, M. R. Tam, and B. H. Weigl, Nature, 442, (2006) 412.

[3] P. S. Dittrich, K. Tachikawa, and A. Manz, Anal. Chem., 78, (2006) 3887.

[4] J. N. Lee, Ch. Park, and G. M. Whitesides, Anal. Chem., $75,(2003) 6544$.

[5] J. C. McDonald, and G. M. Whitesides, Acc. Chem. Res., 35, (2002) 491-499.

[6] Y. Fainman, L. P. Lee, D. Psaltis, and Ch. Yang: "Optofluidics: Fundamentals, Devices, and Application", (McGraw-Hill Professional, San Diego, 2010) p. 7-31.

[7] A. Marcinkevičius, S. Juodkazis, M. Watanabe, M. Miwa, S. Matsuo, and H. Misawa, Opt. Lett., 26, (2001) 277.

[8] Y. Bellouard, A. A. Said, and P. Bado, Opt. Express, 13, (2005) 6635.

[9] K. Sugioka, and Y. Cheng, Lab Chip, 12, (2012) 3576.

[10] G. D. Valle, R. Osellame, and P. Laporta, J. Opt. A: Pure Appl. Opt., 11, (2009) 013001.

[11] K. Sugioka, and Y. Cheng, Light Sci. Appl., 3, (2014) e149.

[12] R. Taylor, C. Hnatovsky, and E. Simova, Laser Photon. Rev., 2, (2008) 26-46. 
[13] C. Hnatovsky, R. S. Taylor, E. Simova, P. P. Rayeev, D. M. Rayner, V. R. Bhardwaj, and P. B. Corkum, Appl. Phys. Mater. Sci. Process., 84, (2006) 47-61.

[14] V. Stankevič, and G. Račiukaitis, J. Laser Micro/Nanoeng., 9, (2014) 271-275.

[15] S. Rekveld, "Ellipsometric Studies of Protein Adsorption onto Hard Surfaces in a Flow Cell", (Ph.D. thesis, Netherlands, 1997).

[16] J. Homola, S. Yee, and G. Gauglitz, Sens. Actuators B, 54, (1999) 3-15.

(Received: June 12, 2015, Accepted: January 17, 2016)
[17] X. Guo, J. Biophotonics, 5, (2012) 483-501.

[18] T. H. Seefeld, A. R. Halpern, and R. M., Corn, J. Am. Chem. Soc., 134, (2012) 12358-12361.

[19] H. Raether, STMP, 111, (2006) 40-57.

[20] M. Poksinski, and H. Arwin, Thin Solid Films, 455456, (2004) 716.

[21] E. P. Diamandis, and T. K. Christopoulos, Clin. Chem., 37, (1991) 625-636. 